\title{
Avaliação de efeito de uma intervenção para a Síndrome de Burnout em professores
}

\author{
Larissa Dalcin', http://orcid.org/0000-0002-8457-2692
}

Mary Sandra Carlotto², http://orcid.org/0000-0003-2336-5224

\section{Resumo}

A Síndrome de Burnout é caracterizada como um estresse crônico que impacta o trabalhador na sua saúde mental e física e requer ações de prevenção e intervenção. Assim, o estudo objetivou avaliar o efeito de uma intervenção para SB em professores. Foi utilizado delineamento pré-experimental com pré e pós-teste. Participaram da intervenção 20 professoras que atuam em uma escola municipal de ensino fundamental da região metropolitana de Porto Alegre, RS. A intervenção constitui-se de seis encontros que abordaram os seguintes temas: autodiagnóstico, estratégias de enfrentamento, manejo de problemas e emoções, gestão do tempo/família $x$ trabalho e expectativas profissionais realísticas no trabalho. Os resultados evidenciaram que as dimensões de ilusão pelo trabalho, coping focado no problema e variabilidade de emoções no trabalho foram as variáveis que obtiveram aumento significativo quando comparados os tempos 1 e 2 de aplicação dos testes.

Palavras-chave: Síndrome de Burnout; trabalho docente; intervenção psicológica.

\section{Evaluation of the effect of an intervention for Burnout Syndrome in teachers}

\begin{abstract}
Burnout Syndrome is characterized by a chronic stress that impacts the worker on his mental and physical health and requires actions of prevention and intervention. Thus, the study aimed to evaluate the effect of an intervention for SB in teachers. A pre-experimental design with pre and post-test was used. Twenty teachers participated in the intervention, working in a municipal elementary school in the metropolitan region of Porto Alegre, RS. The intervention consists of six meetings that dealt with the following themes: self-diagnosis, coping strategies, problem and emotion management, time/family vs. work management and realistic professional expectations at work. The results showed that the dimensions of illusion for work, problem-focused coping, and variability of emotions at work were the variables that obtained a significant increase when compared times 1 and 2 of application of the tests.
\end{abstract}

Keywords: Burnout syndrome; teaching work; psychological intervention.

\section{Evaluación de efecto de una intervención a la Síndrome de Burnout en profesores}

\section{Resumen}

La Síndrome de Burnout se caracteriza como un estrés crónico que impacta el trabajador en su salud mental y física y requiere acciones de prevención e intervención. Así, en el estudio se tuvo por objetivo evaluar el efecto de una intervención para SB en profesores. Se utilizó delineamiento pre-experimental con pre y post-test. Participaron de la intervención 20 profesoras que actúan en una escuela municipal de enseñanza básica de la región metropolitana de Porto Alegre, RS. La intervención se constituyó de seis encuentros que abordaron los siguientes temas: auto diagnóstico, estrategias de enfrentamiento, gestión de problemas y emociones, gestión del tiempo/familia x trabajo y expectativas profesionales realísticas en el trabajo. Los resultados evidenciaron que las dimensiones de ilusión por el trabajo, coping enfocado en el problema y variabilidad de emociones en el trabajo fueron las variables que obtuvieron aumento significativo cuando comparados los tiempos 1 y 2 de aplicación de los testes.

Palabras clave: Síndrome de Burnout; trabajo docente; intervención psicológica.

1 Universidade Regional do Noroeste do Estado do Rio Grande do Sul - ljuí, RS, Brasil.

2 Universidade do Vale do Rio dos Sinos - São Leopoldo, RS, Brasil. 


\section{Introdução}

A Síndrome de Burnout (SB) é considerada uma reação ao estresse ocupacional crônico (Gil-Monte, 2011; Maslach, 1993; Leiter \& Maslach, 2014). Trata-se de uma experiência subjetiva de caráter negativo composta de cognições, emoções, atitudes e comportamentos negativos frente ao trabalho e ao seu papel profissional e às pessoas que necessitam se relacionar no trabalho (Gil-Monte, 2011). Burnout é, indiscutivelmente, um dos tópicos em Psicologia da Saúde Ocupacional mais investigados, uma vez que seus resultados têm confirmado as sérias consequências em termos de saúde dos trabalhadores e organizações (Bakker \& Costa, 2014)

Na perspectiva de Gil-Monte (2005), a SB constitui-se em quatro dimensões: 1) Ilusão pelo trabalho, que traduz o desejo do indivíduo de atingir suas metas de trabalho, assim, fazendo da atividade uma fonte de realização pessoal; 2) Desgaste psíquico, que se refere ao esgotamento físico e emocional ocasionado por ter de lidar diariamente com pessoas que apresentem ou, ainda, que causem problemas; 3) Indolência, caracterizada por atitudes negativas e distanciamento para com seus clientes, demonstrando insensibilidade perante os problemas aos quais necessita atender; 4) Culpa, caracterizada pelo surgimento de sentimentos de culpabilização que a pessoa desenvolve por manifestar atitudes e comportamentos não condizentes com as normas internas e cobranças sociais do seu papel profissional.

A docência é uma profissão que é alvo de diversos estressores psicossociais (Carlotto, 2011). Além de dar aulas, os professores devem executar tarefas administrativas (Gil-Monte, Carlotto, \& Câmara, 2011), lidar com a falta de interesse e a indisciplina dos alunos, a falta de estrutura e recursos, a falta de diálogo e apoio de administradores, gestores públicos e pais (Carlotto \& Palazzo, 2006; Costa \& Rocha, 2013). Também, verifica-se uma crescente falta de reconhecimento social, pois os professores têm trabalhado com uma população de alunos que exige maior dedicação e cuidados, indo muito além das condições e formação do profissional, o que acaba por gerar sobrecarga de papéis e de trabalho (Diehl \& Carlotto, 2014; Reis, Araújo, Carvalho, Barbalho, \& Silva, 2006).

Predominantemente, a literatura procura entender, definir e explicar as variáveis associadas ao Burnout, mas, recentemente, busca, também, propor ações que possam ser aplicadas por profissionais nos diferentes contextos de trabalho. Nesse sentido, verifica-se uma ampliação de estudos sobre intervenção, que visam alinhar a teoria já existente ao desenvolvimento de intervenções específicas (Leiter \& Maslach, 2014).

O estresse no ensino não pode ser eliminado, no entanto, pode ser reduzido a níveis controláveis para que os professores sejam capazes de operacionalizarem suas atividades de forma eficiente mantendo sua a saúde mental. Nesse sentido, é importante capacitar professores sobre temas como equilíbrio entre as demandas do trabalho e vida pessoal, sobre o estabelecimento de metas realistas e como desenvolver estratégias de enfrentamento ao estresse ocupacional (Kourmousi \& Alexopoulos, 2016).
As intervenções devem ser pensadas e elaboradas de acordo com os diferentes níveis em que o fenômeno estudado se manifesta e pelas variáveis que afeta, sendo que estas podem se apresentar em nível macro, meso ou micro (Abbad, Palácios, \& Gondim, 2014). Burnout, geralmente, tem sido estudado em dois grupos de variáveis: 1 . Fatores individuais, que compreendem as variáveis sociodemográficas e de personalidade; e 2. Fatores contextuais relacionados ao cargo/trabalho, que incluem os aspectos da organização e do contexto do trabalho (Boa \& Deps, 2015; Maslach, Schaufeli, \& Leiter, 2001; Nogueira, 2012).

Uma das formas utilizadas pelo indivíduo para a prevenção da síndrome são as estratégias de coping, que são definidas como um esforço cognitivo e comportamental utilizado para diminuir ou tolerar as demandas advindas dos meios interno ou externo (Christofoletti, Trelha, Galera, \& Feracin, 2007; Rodrigues, 2006). Pode-se perceber o poder do coping como preditor de doença, verificando que as estratégias de enfrentamento que são utilizadas pelos indivíduos são decisivas na transição do estresse ao desenvolvimento da Síndrome de Burnout (Sousa, Mendonça, Zanini, \& Nazareno, 2009). O coping pode ser um dos fatores de proteção da SB (Gil-Monte, 2005; Herruzo-Cabrera \& Moriana-Elvira, 2004). Estudos realizados apontam que as estratégias de coping mais utilizadas por professores foram, em primeiro lugar, estratégias focalizadas no problema, seguidas de estratégias de evitação e, por último, estratégias referentes à emoção, além disso, os estudos também revelam que níveis mais baixos de estresse foram avaliados em professores que adotam estratégias de coping centradas no problema (Capelo, 2010; Pocinho \& Capelo, 2009; Quirino, 2007).

O trabalho emocional pode ser visto como um processo individual no qual a gestão das emoções pelos trabalhadores pode prever os níveis de burnout (Hochschild, 1983). Nesta perspectiva, é importante que os trabalhadores saibam gerenciar seus sentimentos para que possam expressar emoções necessárias ao exercício de sua função laboral, mesmo que esse esforço não lhes seja claro (Hochschild, Irwin, \& Ptashne, 1983). O controle emocional é necessário para que se possa manter uma relação positiva com os clientes ao longo do tempo e das situações (Hochschild, 1983; VanMaanen \& Kunda, 1989). Estudo realizado por Philipp e Schüpbach (2010) demonstra que os professores que foram capazes de gerenciar suas emoções para determinadas situações sentiram-se menos exaustos emocionalmente após um ano.

Equilibrar a interação trabalho-família, também, tem sido alvo de estudos, especialmente em mulheres, pois além de desenvolver as atividades da sua vida laboral, elas ainda executam as tarefas familiares, portanto, observa-se que a dupla jornada de trabalho pode ocasionar conflitos (Guimarães \& Petean, 2012). De acordo com Arroyo (2000), poucos trabalhos se identificam tanto com a vida pessoal do trabalhador quanto a do professor. Neste sentido, o trabalho docente é altamente exigente, e acaba por comprometer o tempo de lazer e convívio familiar (Vasques-Menezes \& Gazzotti, 1999). 
Estudo desenvolvido por Carlotto (2014) relata a experiência de uma intervenção psicossocial realizada para a prevenção da SB em professores. A intervenção foi estruturada em cinco sessões realizadas quinzenalmente, nas quais foram trabalhadas temáticas como o autodiagnóstico e a identificação de fatores de risco, desenvolvimento de estratégias saudáveis de enfrentamento de estresse, manejo de problemas, expectativas realistas do trabalho e uma proposta para a prevenção de Burnout. Os resultados revelaram que a intervenção foi benéfica para ampliação do conhecimento sobre Burnout, seus principais fatores de risco e formas de prevenção por meio da troca de vivências entre os participantes.

Outro estudo sobre intervenção, realizado por Żołnierczyk-Zreda (2005), cujo objetivo foi diminuir o Burnout em professores, identificou que o maior efeito da intervenção foi verificado a partir do aumento do controle comportamental sobre o trabalho. O autor conclui, portanto, que propiciar aos os docentes aprendizagem para o gerenciamento dos estressores laborais possibilitou que esses mudassem sua percepção sobre as características estressantes do trabalho, também diminuindo a exaustão emocional e as queixas somáticas.

Os programas de intervenção para diminuição da SB são benéficos, pois as pessoas que participam, comumente, apresentam redução significativa no desgaste ou mudanças positivas em relação aos fatores de risco de Burnout (Awa, Plaumann, \& Walter, 2010). A avaliação de programas de intervenção é importante na medida em que serve de instrumento de controle para avaliar intervenções planejadas e prover informações para a tomada de decisão por parte dos gestores. Nesse sentido, possibilita gerir a manutenção, a modificação ou, em determinados casos, o término de um programa (Murta, Laros, \& Troccoli, 2005).

Pelo exposto, o objetivo deste estudo foi avaliar o impacto de uma intervenção para Síndrome de Burnout em professores. As hipóteses do estudo foram: H1: A intervenção aumenta o índice de llusão pelo trabalho e diminui os índices das dimensões de Desgaste Psicológico, Indolência e Culpa pós-intervenção; H2: A intervenção aumenta o índice de estratégias de coping com foco no problema e diminui as estratégias de coping com foco na emoção e coping de evitação pós-intervenção; H3: A intervenção aumenta os índices de interação trabalho-família positiva e família-trabaIho positiva e diminui a interação trabalho-família negativa e família-trabalho negativa pós-intervenção; H4: A intervenção aumenta os índices de frequência, intensidade, variabilidade e regulação das emoções pós-intervenção.

\section{Método}

\section{Delineamento}

Trata-se de um estudo pré-experimental. Esse tipo de delineamento é utilizado nos estudos que utilizam apenas um único grupo, sem grupo controle, e que seja aplicado pré e pós-teste (Cozby, 2003).

\section{Participantes}

Participaram do estudo 20 professoras que atuam em uma escola de ensino fundamental pública municipal da região metropolitana de Porto Alegre/RS. As participantes foram convidadas a participar da intervenção de forma voluntária, a partir de explanação dos objetivos do grupo em reunião de professores. A maioria das participantes possui companheiro fixo $(57,9 \%)$, filhos $(60 \%)$ e idade média de 42,70 anos (DP=10,28). Quanto à formação, 75\% possuem pós-graduação e, no que diz respeito à remuneração, 70\% recebem de 3 a 6 salários mínimos. Possuem, em média, de 18,37 anos de docência ( $\mathrm{DP}=7,65)$ e 9,12 anos de docência na instituição investigada ( $D P=7,95)$. A maioria trabaIha exclusivamente como professora (80\%) e na escola da investigação (85\%). As profissionais trabalham, em média, 35,30 horas semanais $(\mathrm{DP}=14,19)$ e atendem diariamente, em média, 178,70 alunos $(D P=288,40)$.

\section{Instrumentos}

Os instrumentos autoaplicáveis utilizados para a descrição da amostra e avaliação do efeito da intervenção foram:

1. Questionário de dados sociodemográficos (sexo, idade, estado civil, situação conjugal, filhos) e laborais (formação, carga horária semanal, tempo de experiência profissional, tempo de experiência na atual escola, quantidade de alunos que atende diariamente, trabalha em outra instituição, possui outra atividade profissional).

2. Questionário para avaliação da Síndrome de Burnout - Questionário para a Avaliação da Síndrome de Quemarse por el Trabajo - CESQT-PE (Gil-Monte, 2005), versão adaptada para o uso no Brasil realizada por Gil-Monte, Carlotto e Câmara (2010). O instrumento conta com 20 itens que se distribuem em quatro subescalas denominadas: Ilusão para o trabalho (O meu trabalho representa para mim um desafio estimulante, 5 itens, alfa $=0,72$ ); Desgaste psíquico (Penso que estou saturado/a pelo meu trabalho, 4 itens, alfa $=0,86$ ); Indolência (Não me agrada atender algumas pessoas em meu trabalho, 6 itens, alfa =0,75); e Culpa (Preocupa-me a forma como tratei algumas pessoas no trabalho, 5 itens, alfa $=0,79)$. Os itens são avaliados com uma escala tipo Likert de frequência de cinco pontos (0 "nunca" a 4 "todos os dias").

3. COPE Inventory - Inventário para avaliação das estratégias de Coping - de Carver, Sheier e Weintraub (1989). Esta ferramenta totaliza 60 itens com sistema de pontuação de 1 a 4 , sendo o 1 para "não costumo fazer isso nunca", o 2 para "costumo fazer isso um pouco", o 3 para "costumo fazer isso moderadamente" e o 4 para "costumo fazer isso muito". A escala é constituída de 15 fatores, com quatro itens para cada estratégia avaliada que determinam um perfil de estratégias de coping: o coping ativo, o planejamento, a supressão de atividades concomitantes, o coping moderado, a busca de suporte social por razões instrumentais, a busca de suporte social por razões emocionais, a reinterpretação positiva, a aceitação, o retorno para a religiosidade, o foco na 
expressão das emoções, a negação, o comportamento descomprometido, o desengajamento mental, o humor e o uso de substâncias. Na escala original, a fidedignidade encontrada em relação à consistência interna variou de 0,62 a 0,92 nos 15 fatores. Para a análise dos dados, os 15 fatores de coping foram agrupados em três dimensões de enfrentamento, quais sejam, coping focalizado no problema (tento encontrar uma estratégia sobre o que fazer), coping centrado na emoção (sinto-me angustiado e deixo aflorar minhas emoções) e coping de evitação (concentro-me em outras atividades para afastar o problema da minha mente), agrupamento também realizado em estudos de Dobreva-Martinova, Villeneuve, Strickland e Matherson (2002) e de Litman (2006).

4. Escala de Interação Trabalho-Família - Survey Work-Home Interaction - Nijmegen (SWING) de Geurts et al. (2005), traduzida e adaptada para o Brasil por Carlotto e Câmara (2014). O instrumento consta de 22 itens que se distribuem em quatro subescalas denominadas: Interferência negativa trabalho-família (ITF-) que avalia o impacto negativo de situações de trabalho no funcionamento familiar (Sinto-me irritado em casa por causa das exigências no trabalho) (8 itens, alfa $=0,92$ ); Interferência negativa família-trabalho (IFT-) que mede o impacto negativo de questões familiares na situação de trabalho (Tenho dificuldade em me concentrar no trabalho por estar preocupado com um problema na família) (4 itens, alfa= 0,84); Interferência positiva trabalho-família (ITF+), referindo-se a influência positiva de situações de trabalho no funcionamento familiar (Sinto-me mais capaz de interagir com a família, amigos e cônjuge por casa do que aprendi no trabalho) (5 itens, alfa= 0,84) e Interferência positiva família-trabalho (IFT+) caracterizada pelo impacto positivo no funcionamento familiar de situações positivas ocorridas no trabalho (Depois de passar um agradável fim de semana com a família, amigos e cônjuge, sinto mais satisfação no trabalho.) (5 itens, alfa= 0,81). Os itens são avaliados em uma escala tipo Likert de frequência de cinco pontos ( 1 "nunca" a 5 "sempre").

5. Escala de Emoções no Trabalho - de Brotheridge e Lee (2003), traduzida e adaptada para o Brasil por Carlotto, Rodriguez e Câmara (2016) para a avaliação do trabalho emocional. O instrumento contém 15 itens distribuídos em quatro subescalas denominadas: Frequência (expresso emoções específicas exigidas por meu trabalho) (3 itens, alfa $=0,67$ ); Intensidade (expresso emoções intensas no trabalho) (2 itens, alfa $=0,78$ ); Variabilidade (expresso muitos tipos diferentes de emoções no trabalho) (3 itens, alfa $=0,84$ ) e Regulação da emoção (procuro expressar meus verdadeiros sentimentos no trabalho) (4 itens, alfa $=0,79$ ). Os itens são avaliados a partir de uma escala tipo Likert de frequência de quatro pontos (0 "nunca" a 3 "sempre").

\section{Intervenção}

A intervenção foi realizada mensalmente, em seis encontros com 2 horas de duração, totalizando 12 horas, em uma sala de aula da escola. A condução das sessões foi desenvolvida pela primeira autora do estudo, a partir de duas estratégias, presencial, por meio de oficinas, e a distância, por meio de material disponibilizado via e-mail.

As oficinas possibilitam que o indivíduo desenvolva habilidades fundamentais como a escuta, o enfrentamento de situações de estresse e o aperfeiçoamento do diálogo (Graças Nascimento, Melo, \& Silva, 2014). Segundo Abbad e Borges-Andrade (2004), a capacitação dos profissionais pode ocorrer de diferentes formas. O desenvolvimento e a utilização das novas tecnologias de informação e comunicação (NTICs) em treinamentos estão se tornando essenciais para facilitar a aquisição e atualização de conhecimentos dos indivíduos (Zerbini \& Abbad, 2005). Portanto, entre os intervalos de um encontro e outro, foram enviados e-mails para o grupo de professores participantes da intervenção que continham vídeos e textos com conteúdos do que haviam sido apresentados e discutidos no encontro anterior a fim de dar continuidade ao trabalho. A descrição de cada encontro está apresentada na Figura 1.

\section{Procedimentos de coleta de dados}

Para a coleta de dados, primeiramente, foi realizado contato com a Secretaria de Educação do município e, posteriormente, com a instituição de ensino para apresentação do objetivo do estudo com intuito de obter a autorização e o apoio para a aplicação dos instrumentos e realização da intervenção. Os questionários foram respondidos pelos participantes do programa de intervenção em espaço cedido na reunião mensal de professores, no mês de maio de 2015 (pré-teste) e no mês de outubro de 2015 (pós-teste). Após o preenchimento, os questionários foram devolvidos à pesquisadora.

\section{Procedimentos de Análise}

O banco de dados foi digitado e, posteriormente, analisado em pacote estatístico Statistical Package for the Social Sciences (SPSS), versão 17.0. Primeiro, foram realizadas análises descritivas de caráter exploratório a fim de avaliar, no banco de dados, a distribuição dos itens, os casos omissos, a identificação de extremos e a descrição dos participantes. Após, foi realizada a comparação dos resultados entre o pré-teste (T1) e o pós-teste (T2), por meio do teste estatístico não paramétrico Wilcoxon Signed-Rank, para amostras pareadas. Foi adotado o nível de significância de $p<0,05$.

\section{Considerações éticas}

O estudo teve aprovação do Comitê de Ética em Pesquisa da PUCRS. Todos os professores preencheram e assinaram o Termo de Consentimento Livre e Esclarecido. 


\begin{tabular}{|c|c|c|c|}
\hline Módulo & Temática & Objetivo & T écnica \\
\hline 1 & $\begin{array}{l}\text { Avaliação Pré-teste } \\
\text { Contrato de trabalho } \\
\text { SB/autodiagnóstico }\end{array}$ & $\begin{array}{l}\text { Fomecer informações sobre a SB, } \\
\text { suas dimensões, principais } \\
\text { caracteristicas, sintomas e suas } \\
\text { consequências. Estimular o } \\
\text { reconhecimento e a existência dos } \\
\text { estressores presentes na profissão } \\
\text { docente }\end{array}$ & $\begin{array}{l}\text { Exposição teórica. } \\
\text { Atividade prática de } \\
\text { reconhecimento de estressores. } \\
\text { Tarefa de casa: Escreva sua } \\
\text { própria narrativa de trabalho. }\end{array}$ \\
\hline 2 & $\begin{array}{l}\text { Desenvolvimento de } \\
\text { estratégias saudáveis } \\
\text { de enfrentamento }\end{array}$ & $\begin{array}{l}\text { Desenvolver estratégias saudáveis } \\
\text { para lidar com demandas } \\
\text { especificas, interna ou extemas que } \\
\text { surgem em situações de estresse. }\end{array}$ & $\begin{array}{l}\text { Exposição teórica. } \\
\text { Atividade prática de } \\
\text { reconhecimento dos } \\
\text { estressores laborais e } \\
\text { estratégias de enfrentamento. } \\
\text { Tarefa de casa: Adaptar as } \\
\text { estratégias saudáveis no seu } \\
\text { dia adia. }\end{array}$ \\
\hline 3 & $\begin{array}{l}\text { Manejo de problemas } \\
\text { e emoções }\end{array}$ & $\begin{array}{l}\text { Desenvolver capacidade de análise } \\
\text { da situação para estruturação de } \\
\text { plano estratégico e efetivo de } \\
\text { resolução de problemas, expressar e } \\
\text { avaliar, de forma adequada, as } \\
\text { emoções, identificar seus } \\
\text { significados, compreendendo as } \\
\text { informações nelas contidas de } \\
\text { maneira a conseguir administrá-las. }\end{array}$ & $\begin{array}{l}\text { Exposição teórica. } \\
\text { Atividade prática de } \\
\text { construção de problemas. } \\
\text { Tarefa de casa: O que tem no } \\
\text { seu relógio? }\end{array}$ \\
\hline 4 & $\begin{array}{l}\text { Gestão do tempo/ } \\
\text { trabalho, familia }\end{array}$ & $\begin{array}{l}\text { Propiciar o desenvolvimento de } \\
\text { habilidades para estabelecer } \\
\text { prioridades frente a situações } \\
\text { laborais de urgência, gerenciamento } \\
\text { entre atividade de trabalho e familia. } \\
\text { Refletir sobre o conceito de familia } \\
\text { e sua importância para o equilibrio } \\
\text { dinâmico do profissional, suas } \\
\text { dimensões fisica, emocional, social, } \\
\text { ambiental e espiritual. }\end{array}$ & $\begin{array}{l}\text { Exposição teórica. } \\
\text { Atividade prática de Aprendaa } \\
\text { dizer não (ou sim)! } \\
\text { Tarefa de casa: Prática de } \\
\text { lazer. }\end{array}$ \\
\hline 5 & $\begin{array}{l}\text { Expectativas } \\
\text { profissionais } \\
\text { realisticas } \\
\text { participação dos pais } \\
\text { na escola }\end{array}$ & $\begin{array}{l}\text { Discutir as crenças acerca de sua } \\
\text { prática, auxiliando a desenvolver } \\
\text { concepções mais realisticas e } \\
\text { adequadas à profissão. Atenuar o } \\
\text { sentimento de responsabilização e } \\
\text { vitimização para os estressores } \\
\text { presentes no contexto de trabalho. }\end{array}$ & $\begin{array}{l}\text { Exposição teórica } \\
\text { Atividade brainstorming. }\end{array}$ \\
\hline 6 & $\begin{array}{l}\text { Avaliação Pós-teste } \\
\text { Avaliação oral }\end{array}$ & $\begin{array}{l}\text { Levantar propostas individuais, } \\
\text { grupais e organizacionais para a } \\
\text { prevenção da SB. Encerramento e } \\
\text { avaliação oral da intervenção. }\end{array}$ & Pós/teste e encerramento \\
\hline
\end{tabular}

Figura 1: Descrição das sessões.

\section{Resultados}

A Tabela 1 apresenta as médias, desvios padrão e os resultados obtidos por meio de teste estatístico para comparar os escores do grupo nos tempos 1 e 2 , a partir das escalas de SB, coping, interação trabalho-família e emoções no trabalho. A llusão pelo trabalho foi a dimensão de Burnout que apresentou resultado significativo quando avaliada a diferença entre os dois tempos. Em relação às dimensões de coping, observou-se que o coping focalizado no problema 
foi a única dimensão que obteve resultado estatisticamente significativo, quando comparados os tempos 1 e 2. Os resultados obtidos sobre as emoções no trabalho demonstram que a variabilidade de emoções apresentou escore estatisticamente significativo.

\section{Discussão}

O estudo teve por objetivo avaliar o impacto de uma intervenção para Síndrome de Burnout aplicada em professores. Os resultados confirmaram parcialmente as hipóteses do estu- do, uma vez que somente a llusão pelo trabalho, o coping focalizado no problema e a variabilidade de emoções apresentaram aumento significativo na avaliação pré e pós-intervenção.

A llusão pelo trabalho refere-se às expectativas que o indivíduo tem em alcançar determinadas metas laborais que se configuram em maior realização pessoal e profissional (Gil-Monte, Carlotto, \& Câmara, 2010). Ainda, de acordo com os autores, diferentemente das demais subescalas do CESQT, os valores altos, quando obtidos em llusão para o trabalho, podem ser visualizados como positivos, pois altas pontuações nesta variável referem-se ao indicativo de baixos níveis de SB.

Tabela 1. Resultados da avaliação.

\begin{tabular}{|c|c|c|c|}
\hline & Tempo 1 & T empo 2 & Wilcoxon \\
\hline & $\mathrm{M}(\mathrm{DP})$ & $\mathrm{M}(\mathrm{DP})$ & Signed-Rank \\
\hline \multicolumn{4}{|l|}{ Bumout } \\
\hline Ilusão & $2,87(0,91)$ & $3,39(0,64)$ & $0,02 *$ \\
\hline Desgaste Psíquico & $1,96(0,93)$ & $2,15(0,88)$ & 0,36 \\
\hline Indolência & $1,27(0,71)$ & $2,14(0,94)$ & 0,08 \\
\hline Culpa & $1,17(0,60)$ & $1,33(0,85)$ & 0,13 \\
\hline \multicolumn{4}{|l|}{ Coping } \\
\hline Coping focado no problema & $2,55(0,59)$ & $2,99(0,48)$ & $0,01 *$ \\
\hline Coping focado na emoção & $2,55(0,41)$ & $2,57(0,33)$ & 0,93 \\
\hline Coping de evitação & $1,70(0,35)$ & $1,77(0,45)$ & 0,96 \\
\hline \multicolumn{4}{|l|}{ Interação trabalho- família } \\
\hline Trabalho - família negativa & $1,17(0,60)$ & $1,18(0,61)$ & 0,98 \\
\hline Família - trabalho negativa & $0,86(0,49)$ & $0,78(0,70)$ & 0,42 \\
\hline Trabalho - família positiva & $1,43(0,69)$ & $1,41(0,80)$ & 0,85 \\
\hline Família - trabalho positiva & $1,58(0,89)$ & $1,76(0,91)$ & 0,28 \\
\hline \multicolumn{4}{|l|}{ Emoções no trabalho } \\
\hline Frequência & $3,03(0,83)$ & $3,36(0,75)$ & 0,08 \\
\hline Intensidade & $2,52(1,04)$ & $2,92(0,86)$ & 0,06 \\
\hline Variabilidade & $2,60(0,95)$ & $3,03(0,89)$ & $0,05 *$ \\
\hline Regulação & $2,58(0,91)$ & $2,91(0,73)$ & 0,17 \\
\hline
\end{tabular}

Nota: * $p<0,05$ 
Esse resultado confirma a primeira hipótese do estudo, ou seja, que a intervenção aumentaria o índice de llusão pelo trabalho. Isso pode ser entendido como derivado da proposta da quinta sessão, que trabalhou as expectativas realísticas em relação ao trabalho na medida em que objetivou a discussão sobre o papel docente no atual contexto educacional, suas possibilidades e seus limites.

Duas questões podem explicar esse resultado, a primeira diz respeito à formação docente, pois, segundo Rudow (1999), muitas vezes, os professores têm de conviver com uma realidade diferente da que Ihes foi apresentada na sua formação, constatando que a teoria se apresenta diferente da prática. A segunda diz respeito ao sentimento de responsabilização e autocobrança docente. Professores se sentem obrigados a responder às novas demandas pedagógicas e administrativas decorrentes das reformas educacionais que se concretizam no cotidiano escolar com impacto no seu trabalho, relações interpessoais e identidade profissional. Esse processo faz com que os profissionais se autorresponsabilizem pelas falhas ocorridas na escolarização de seus alunos, pelos seus problemas sociais e por outros problemas que surgem na escola (Duarte, Oliveira, Augusto, \& Melo, 2008). A interação entre as características laborais e as expectativas do sujeito pode constituir-se em fonte de estresse (Queirós, 2005), que, por sua vez, pode conduzir ao Burnout (Forte, 2009; Kim, Lee, \& Kim, 2009; Oliveira \& Guerra, 2004).

O resultado relativo a não diferença significativa dos índices de Burnout nas outras dimensões avaliadas: Desgaste Psíquico, Indolência e Culpa, sugere o risco psicossocial ao qual esses profissionais estão expostos no seu contexto laboral e que não pôde ser modificado por meio da intervenção. Apesar de intervenção ter sido realizada em nível individual, pode-se pensar na presença de estressores contextuais, pouco manejáveis pelo professor, como baixos salários, inexpressiva participação nas políticas e planejamento institucional (Melo, Rego, Saldanha, Oliveira, \& Maracaja, 2015).

Quanto ao coping, o resultado obtido confirmou parcialmente a segunda hipótese do estudo, que refere que a intervenção aumenta o índice de estratégias de coping com foco no problema e diminui as estratégias de coping com foco na emoção e coping de evitação pós-intervenção, considerando que somente o coping focado no problema apresentou índices significativamente mais elevados pós-intervenção. $O$ resultado pode sinalizar o impacto da intervenção sobre a forma de manejar estressores, assim, buscando atuar na situação que deu origem ao estresse, no sentido de alterá-la (Folkman \& Lazarus, 1980). Esse resultado é importante na medida em que indivíduos que utilizam com maior frequência estratégias centradas no problema possuem maior capacidade de prevenir o Burnout (Carmona, Buunk, Peiró, Rodríguez, \& Bravo, 2006; Gil-Monte, 2005).

Também foi confirmada parcialmente a hipótese 4 do estudo, que postulava que a intervenção aumentaria os índices de frequência, intensidade, variabilidade e regulação das emoções pós-intervenção. O efeito da intervenção foi verificado somente na dimensão de variabilidade, assim, indicando que os professores passaram a utilizar maior diversidade de expressões emocionais no contexto do seu trabalho, possivelmente, em relação aos alunos. Este resultado pode ser derivado da proposta da terceira sessão, que trabalhou o manejo de emoções e teve por objetivo identificar as emoções e seus significados, bem como, expressar e avaliar de forma adequada as emoções em seu cotidiano laboral, buscando compreende-las a fim de conseguir administrá-las.

Além disso, acredita-se que a diversidade de expressões emocionais pode levar o indivíduo a uma maior realização profissional, pois este consegue gerir suas emoções, expressando-as de forma positiva ou negativa, de acordo com o esperado pela instituição, mas de forma autônoma. Essa dimensão está associada a um aumento da empatia e simpatia nas quais o profissional presta atendimento que, por sua vez, ocasionaria maior sentimento de eficácia e realização profissional (Brotheridge \& Grandey, 2002).

\section{Conclusão}

O presente estudo apresentou a avaliação do impacto de um programa de intervenção para a Síndrome de Burnout em professores. Por meio dos resultados obtidos, pôde-se concluir que a intervenção confirmou parcialmente as hipóteses do estudo, considerando que somente a ilusão pelo trabalho, o coping focalizado no problema e a variabilidade das emoções no trabalho apresentaram aumento significativo na avaliação pré e pós-intervenção.

Nesse sentido, a intervenção mostrou impacto em importantes dimensões que previnem a SB no grupo investigado, pois os professores aumentaram o desejo de alcançar suas metas e avaliar seu trabalho como fonte de realização, passaram a experimentar novas formas de lidar com os estressores, buscando soluções focalizadas no problema, e começaram a utilizar maior diversidade de emoções positivas no trabalho.

O estudo apresenta algumas limitações que devem ser consideradas na análise dos seus resultados. A primeira é a não utilização de grupo controle, não sendo possível afirmar se as mudanças que ocorreram na comparação entre T1 e T2 são exclusivamente decorrentes da intervenção. A segunda seria o número reduzido de participantes, visto que, possivelmente, profissionais acometidos ou mais suscetíveis ao desenvolvimento de Burnout não tenham participado da intervenção, principalmente por perceberem esta como mais um fator de estresse.

É importante destacar que se trata de um primeiro estudo que deve, necessariamente, ser replicado para a consolidação dos resultados obtidos. Assim, para futuros estudos, sugere-se a inclusão de novas técnicas para a intervenção voltadas para as variáveis que não apresentaram resultados significativos. Recomenda-se, também, que seja realizado follow-up, além das avaliações pré e pós-intervenção (Awa \& cols., 2010), para o acompanhamento dos resultados do impacto da intervenção. 
A avaliação contínua das intervenções de saúde no trabalho é essencial para o refinamento da teoria, validação e desenvolvimento de estratégias baseadas em evidências (Biggs, Brough, \& Barbour, 2014). Dessa forma, podem auxiliar gestores e trabalhadores no melhor gerenciamento dos riscos psicossociais presentes no contexto de trabalho. Os resultados obtidos no presente estudo podem, mesmo que ainda incipientes, caracterizar-se como um importante avanço para a Psicologia da Saúde Ocupacional no que diz respeito a seu foco voltado para a pesquisa e aplicação prática de seus conhecimentos.

\section{Referências}

Abbad, G.S.; Borges-Andrade, J.E. (2004). Aprendizagem humana em organizações e trabalho. Psicologia, Organizações e Trabalho no Brasil. Porto Alegre: Artmed.

Abbad, G.; Palácios, K.P.; Gondim, S.M.G. (2014). Abordagens metodológicas em psicologia organizacional e do trabalho. Revista Brasileira de Psicologia, 2 (2), 71-88.

Arroyo, M.G. (2000). Ofício de mestre: imagens e auto-imagens. Rio de Janeiro: Vozes.

Awa, W.L.; Plaumann, M.; Walter, U. (2010). Burnout prevention: a review of intervention programs. Patient Education and Counseling, 78(2), 184-190.

Bakker, A.B.; Costa, P.L. (2014). Chronic job burnout and daily functioning: a theoretical analysis. Burnout Research, 1(3), 112-119.

Biggs, A.; Brough, P.; Barbour, J. P. (2014). Strategic alignment with organizational priorities and work engagement: a multi-wave analysis. Journal of Organizational Behavior, 35(3), 301-317.

Boa, S.V.D.R.; Deps, V.L. (2015). Prevenção e tratamento do estresse e da Síndrome de Burnout em professores da rede pública de ensino. LINKSCIENCEPLACE - Revista Científica Interdisciplinar, 2(1), 62-75.

Brotheridge, C.M.; Grandey, A.A. (2002). Emotional labor and burnout: comparing two perspectives of "people work". Journal of Vocational Behavior, 60(1), 17-39.

Brotheridge, C.M.; Lee, R.T. (2003). Development and validation of the emotional labour scale. Journal of Occupational and Organizational Psychology, 76(3), 365-379.

Capelo, M.R. (2010). Estrés, coping y autoeficacia en profesores de Madeira. (Tese de doutorado). Universidad de Cádiz, Cádiz - Espanha.

Carlotto, M.S. (2011). Síndrome de Burnout em professores: prevalência e fatores associados. Psicologia: Teoria e Pesquisa, 27(4), 403-410.
Carlotto, M.S. (2014). Prevenção da Síndrome de Burnout em professores: um relato de experiência. Mudanças - Psicologia da Saúde, 22(1), 31-39.

Carlotto, M.S.; Câmara, S.G. (2014). Tradução, adaptação e exploração de propriedades psicométricas da Escala Interação Trabalho-Família Nijmen (SWING) em uma amostra de professores brasileiros. Estudos de Psicologia, 19(3), 210-216.

Carlotto M. S.; Palazzo LS. (2006). Síndrome de Burnout e fatores associados: um estudo epidemiológico com professores. Cadernos de Saúde Pública 22(5): 1017-26.

Carlotto, M.S.; Rodriguez, S.I.S.; Câmara, S.G. (2016). Translation, adaptation and exploration of psychometric properties of the Emotional Labor Scale (ELS) in a Sample of Psychologists. Temas de Psicologia 24(2),717-725.

Carmona, C.; Buunk, A.P.; Peiró, J.M.; Rodríguez, I.; Bravo, M. J. (2006). Do social comparison and coping styles play a role in the development of burnout? Cross-sectional and longitudinal findings. Journal of Occupational and Organizational Psychology, 79(1), 85-99.

Carver, C.S.; Scheier, M.F.; Weintraub, J.K. (1989). Assessing coping strategies: a theoretically based approach. Journal of Personality and Social Psychology, 56(2), 267-283.

Christofoletti, G.; Trelha, C.S.; Galera, R.M.; Feracin, M.A. (2007). Síndrome de Burnout em acadêmicos de fisioterapia. Fisioterapia e Pesquisa, 14(2), 35-39.

Costa, F.R.C.P.; Rocha, R. (2013). Fatores estressores no contexto de trabalho docente. Revista Ciências Humanas, 6(1), 18-43.

Cozby, P. (2003). Métodos de pesquisa em ciências do comportamento. São Paulo: Atlas.

Dobreva-Martinova, T.; Villeneuve, M.; Strickland, L.; Matheson, K. (2002). Occupational role stress in the Canadian forces: Its association with individual and organizational well-being. Canadian Journal of Behavioural Science/Revue Canadienne des Sciences du Comportment, 34(2), 111-121.

Diehl, L.; Carlotto, M. S. (2014). Conhecimento de professores sobre a Síndrome de Burnout: processo, fatores de risco e consequências. Psicologia em Estudo, 19(4), 741-752.

Duarte, A.; Oliveira, D.A.; Augusto, M.H.; Melo, S. (2008). Envolvimento docente na interpretação do seu trabalho: uma estratégia metodológica. Cadernos de Pesquisa, 38(133), 221-236.

Folkman, S.; Lazarus, R.S. (1980). An analysis of coping in a middleaged community sample. Journal of Health and Social Behavior, 21(3), 219-239.

Forte, A.I.D.S.C. (2009). Burnout, inteligência emocional e autoatualização em enfermeiros psiquiátricos. (Dissertação de 
Mestrado). Faculdade de Psicologia e de Ciências da Educação: Universidade do Porto, Porto- Portugal.

Geurts, S.; Taris, T.; Kompier, M.; Dikkers, J.; Hooff, M.; Kinnunnen, U. (2005). Workhome interaction from a work psychological perspective: development and validation of a new questionnaire, the SWING. Work \& Stress, 19(4), 319-339.

Gil-Monte, P.R. (2005). El síndrome de quemarse por el trabajo (Burnout): Una enfermidad laboral en la sociedad del bienestar. Madri, España: Ediciones Pirámide.

Gil-Monte, P. R. (2011). CESQT. Cuestionario para la Evaluación Del Síndrome de Quemarse por el Trabajo [SBI. Spanish Burnout Inventory]. Madrid, Spain: TEA.

Gil-Monte, P.R.; Carlotto, M.S.; Câmara, S. (2010). Validation of the Brazilian version of the "Spanish Burnout Inventory" in teachers. Revista de Saúde Pública, 44(1), 140-147.

Gil-Monte, P.R.; Carlotto, M.S.; Gonçalves Câmara, S. (2011). Prevalence of burnout in a sample of Brazilian teachers. The European Journal of Psychiatry, 25(4), 205-212.

Graças Nascimento, L.; Melo, W.; Silva, M.V. (2014). Oficinas de intervenção psicossocial com agentes comunitários de saúde: reflexões e intervenções dialogadas. Revista Brasileira de Medicina de Família e Comunidade, 9(33), 336-342.

Guimarães, M.D.G.V.; Petean, E.B.L. (2012). Carreira e família: divisão de tarefas domiciliares na vida de professoras universitárias. Revista Brasileira de Orientação Profissional, 13(1), 103-110.

Herruzo-Cabrera, J.; Moriana-Elvira, J.A. (2004). Estrés y burnout en profesores. International Journal of Clinical and Health Psychology, 4(3), 597-621.

Hochschild, A.R. (1983). The managed heart. Berkeley, California: University of California Press.

Hochschild, A.; Irwin, N.; Ptashne, M. (1983). Repressor structure and the mechanism of positive control. Cell, 32(2), 319-325.

Kim, M.Y.; Lee, J.Y.; Kim, J. (2009). Relationships among burnout, social support, and negative mood regulation expectancies of elementary school teachers in Korea. Asia Pacific Education Review, 10(4), 475-482.

Kourmousi, N.; Alexopoulos, E.C. (2016). Stress sources and manifestations in a nationwide sample of pre-primary, primary, and secondary educators in Greece. Frontiers Public Health, 4, 73.

Leiter, M.P.; Maslach, C. (2014). Interventions to prevent and alleviate burnout. Burnout at work: a psychological perspective. New York: Psychology Press.

Litman, J.A. (2006). The COPE inventory: Dimensionality and relationships with approach and avoidance-motives and positive and negative traits. Personality and Individual Differences, 41(2), 273-284.

Maslach, C. (1993). Burnout: a multidimensional perspective. IN: Schaufeli, W.B.; Maslach, C.; Marek, T. (Orgs.), Professional burnout: Recent developments in theory and research (pp. 19-32). Washington, DC: Taylor \& Francis.

Maslach, C.; Schaufeli, W.B.; Leiter, M.P. (2001). Job Burnout. Annual Review of Psychology, 52(1), 397-422.

Melo, W.F.; Rego, S.M.D.O.; Saldanha, H.G.A.C.; Oliveira, M.D.F.P.C.; Maracaja, P.B. (2015). Síndrome de burnout em professores. Revista Brasileira de Educação e Saúde, 5(4), 01-06.

Murta, S.G.; Laros, J.A.; Troccoli, B.T. (2005). Manejo de estresse ocupacional na perspectiva da área de avaliação de programas. Estudos de Psicologia, 10(2), 167-176.

Nogueira, I.M.G.D.M. (2012). Clima de escola. (Dissertação de Mestrado). Universidade Fernando Pessoa, Porto, Portugal.

Oliveira, M.; Guerra, M. P. (2004) Burnout nos profissionais de saúde mental: Expectativas, auto-atualização e outras variáveis associadas. Revista Saúde Mental, 4(1), 15-25.

Philipp, A.; Schüpbach, H. (2010). Longitudinal effects of emotional labour on emotional exhaustion and dedication of teachers. Journal of Occupational Health Psychology, 15(4), 494-504.

Pocinho, M.; Capelo, M. R. (2009). Vulnerabilidade ao stress, estratégias de coping e auto-eficiência em professores portugueses. Educação e Pesquisa, 35(2), 351-367.

Queirós, P.J. (2005). Burnout no trabalho conjugal em enfermeiros portugueses. Coimbra, Portugal: Edições Sinais Vitais.

Quirino, A. (2007). Stress, coping e burnout em professores do $3^{\circ}$ ciclo. Dissertação de Mestrado, Universidade do Algarve, Faro, Portugal.

Reis, E.J.F.B.; Araújo, T.M.D.; Carvalho, F.M.; Barbalho, L.; Silva, M.O. (2006). Docência e exaustão emocional. Educação e Sociedade, 27(94), 229-253.

Rodrigues, A.B. (2006). Burnout e estilos de coping em enfermeiros que assistem pacientes oncológicos. (Tese de Doutorado). Universidade de São Paulo, São Paulo, São Paulo.

Rudow, B. (1999). Stress and burnout in the teaching profession: European studies, issues, and research perspectives. IN: Vanderbergue, R.; Huberman, M. A. (Orgs.), Understanding and preventing teacher burnout: a source book of international practice and research (pp. 3858). Cambridge, Londres: Cambridge University Press.

Sousa, I.F.D.; Mendonça, H.; Zanini, D.S.; Nazareno, E. (2009). Estresse ocupacional, coping e burnout. Revista Estudos, 36(1), 57-74. 
VanMaanen, J.; Kunda, G. (1989). Real feelings-emotional expression and organizational culture. Research in Organizational Behavior, 11, 43-103.

Vasques-Menezes, I.; Gazzotti, A.A. (1999). A si mesmo como trabalho. IN: Codo, W. (Org.), Educação: Carinho e Trabalho (pp. 368-383). Petrópolis, RJ: Vozes.
Zerbini, T.;\& Abbad, G. (2005). Impacto de treinamento no trabalho via internet. RAE-eletrônica, 4(2). 1-22.

Żołnierczyk-Zreda, D. (2005). An intervention to reduce work-related burnout in teachers. International Journal of Occupational Safety and Ergonomics, 11(4), 423-430.

Recebido em: 20/12/2016

Aprovado em: 08/12/2017

\section{Sobre as autoras}

Larissa Dalcin (Iddalcin@gmail.com)

Psicóloga, Mestre em Psicologia (PUCRS) e Doutoranda em Educação nas Ciências. Universidade Regional do Noroeste do Estado do Rio Grande do Sul (UNIJUÍ). ljui- RS. http://orcid.org/0000-0002-8457-2692

Mary Sandra Carlotto (mscarlotto@pesquisador.cnpq.br)

Psicóloga, Mestre em Saúde Coletiva (ULBRA); Doutora em Psicologia Social (USC-ES); Programa de Pós-Graduação em Psicologia- Escola de Saúde - Universidade do Vale do Rio dos Sinos - Av. Unisinos, 950 - Cristo Rei, São Leopoldo - RS. http://orcid.org/0000-0003-2336-5224 unrestricted use, distribution and reproduction in any medium, provided the original article is properly cited. 